\title{
Characterization of repetitive DNA landscape in wheat homeologous group 4 chromosomes
}

Ingrid Garbus ${ }^{1 \dagger}$, José R Romero ${ }^{1 \dagger}$, Miroslav Valarik ${ }^{2}$, Hana Vanžurová ${ }^{2}$, Miroslava Karafiátová2 ${ }^{2}$ Mario Cáccamo ${ }^{3}$, Jaroslav Doležel ${ }^{2}$, Gabriela Tranquilli ${ }^{4}$, Marcelo Helguera ${ }^{5}$ and Viviana Echenique ${ }^{\text {* }^{*}}$

\begin{abstract}
Background: The number and complexity of repetitive elements varies between species, being in general most represented in those with larger genomes. Combining the flow-sorted chromosome arms approach to genome analysis with second generation DNA sequencing technologies provides a unique opportunity to study the repetitive portion of each chromosome, enabling comparisons among them. Additionally, different sequencing approaches may produce different depth of insight to repeatome content and structure. In this work we analyze and characterize the repetitive sequences of Triticum aestivum cv. Chinese Spring homeologous group 4 chromosome arms, obtained through Roche 454 and Illumina sequencing technologies, hereinafter marked by subscripts 454 and I, respectively. Repetitive sequences were identified with the RepeatMasker software using the interspersed repeat database mips-REdat_v9.0p. The input sequences consisted of our $4 \mathrm{DS}_{454}$ and $4 \mathrm{DL}_{454}$ scaffolds and $4 \mathrm{AS}$ |, $4 \mathrm{AL} \mathrm{L}_{1}, 4 \mathrm{BS}$, $4 \mathrm{BL}_{\text {I, }}$ 4DS, and 4DL, contigs, downloaded from the International Wheat Genome Sequencing Consortium (IWGSC).

Results: Repetitive sequences content varied from 55\% to 63\% for all chromosome arm assemblies except for 4DL, in which the repeat content was 38\%. Transposable elements, small RNA, satellites, simple repeats and low complexity sequences were analyzed. SSR frequency was found one per 24 to $27 \mathrm{~kb}$ for all chromosome assemblies except $4 \mathrm{DL}$, where it was three times higher. Dinucleotides and trinucleotides were the most abundant SSR repeat units. $(G A) n /(T C) n$ was the most abundant SSR except for $4 D L_{1}$ where the most frequently identified SSR was (CCG/CGG)n. Retrotransposons followed by DNA transposons were the most highly represented sequence repeats, mainly composed of CACTA/En-Spm and Gypsy superfamilies, respectively. This whole chromosome sequence analysis allowed identification of three new LTR retrotransposon families belonging to the Copia superfamily, one belonging to the Gypsy superfamily and two TRIM retrotransposon families. Their physical distribution in wheat genome was analyzed by fluorescent in situ hybridization (FISH) and one of them, the Carmen retrotransposon, was found specific for centromeric regions of all wheat chromosomes.

Conclusion: The presented work is the first deep report of wheat repetitive sequences analyzed at the chromosome arm level, revealing the first insight into the repeatome of T. aestivum chromosomes of homeologous group 4.
\end{abstract}

\section{Background}

Wheat (Triticum aestivum $\mathrm{L}$. em Thell, $2 \mathrm{n}=42$; AABBDD) has an allohexaploid genome structure that arose from two polyploidization events. The first brought together the genomes of two diploid species related to the wild species Triticum urartu $\left(2 \mathrm{n}=2 \mathrm{x}=14 ; \mathrm{A}^{\mathrm{u}} \mathrm{A}^{\mathrm{u}}\right)$ and a species related to Aegilops speltoides $(2 \mathrm{n}=14$; SS) [1]. This hybridization

\footnotetext{
* Correspondence: echeniq@criba.edu.ar

${ }^{\dagger}$ Equal contributors

'CERZOS (CCT - CONICET Bahía Blanca) and Universidad Nacional del Sur, Bahía Blanca, Argentina

Full list of author information is available at the end of the article
}

formed the allotetraploid Triticum turgidum $(2 \mathrm{n}=4 \mathrm{x}=28$; $\mathrm{AABB}$ ) that suffered the second hybridization event with a diploid grass species, Aegilops tauschii (DD), producing the ancestral allohexaploid T. aestivum $(2 \mathrm{n}=6 \mathrm{x}=42$; AABBDD) [1]. Thus, the hexaploid wheat genome is characterized by its large size ( $\sim 17 \mathrm{~Gb})$ and complexity, with repetitive sequences accounting for $\sim 80 \%$ of the genome $[2,3]$.

The number and complexity of repetitive elements varies between species, and those with larger genomes generally have more repetitive elements [4]. Repetitive sequences can be divided into three main classes:

\section{() Biomed Central}

(c) 2015 Garbus et al.; licensee BioMed Central. This is an Open Access article distributed under the terms of the Creative Commons Attribution License (http://creativecommons.org/licenses/by/4.0), which permits unrestricted use, distribution, and reproduction in any medium, provided the original work is properly credited. The Creative Commons Public Domain Dedication waiver (http://creativecommons.org/publicdomain/zero/1.0/) applies to the data made available in this article unless otherwise stated. 
transposable elements, tandem repeats, and high copy number genes, such as ribosomal or histone genes. Transposable elements (TEs) are the best-defined class and constitute the most abundant component of many genomes, ranging from $10 \%$ to $85 \%$ [5]. Based on transposition mechanism, TEs can be subdivided into two classes. Class I, retrotransposons, move via so-called "copy and paste" mechanisms using RNA intermediates, and is mainly composed of long terminal repeat (LTR) retrotransposons and non-LTR retrotransposons, such as LINEs and SINEs (long and short interspersed nuclear elements, respectively) [6]. Class II DNA transposons replicate without an RNA intermediate, either by a cut-and-paste mechanism (terminal inverted repeats; TIRs), by rolling-circle DNA replication (helitrons), or by mechanisms that remain unknown $[5,6]$.

Tandem repeats represent a second class of repetitive sequences that can account for a large portion of genomic DNA, comprising any sequence found in consecutive copies along a DNA strand, arranged in tandem arrays of the monomeric unit [7]. Typically localized to specialized chromosome regions such as centromeres, telomeres, and heterochromatic knobs of many eukaryotes [8], tandem repeats can be categorized according to the size of the repeated units. Microsatellites or simple sequence repeats consist of 1-6 nucleotides, minisatellites are 10-60 nucleotides, and satellites include more than 60 nucleotides. Satellites are the main class of tandem repeats and are thought to play a role in organizing and stabilizing the specialized chromosome regions in which they are found, which are important for chromosome behavior during cell division [9]. Whereas some satellite repeats are chromosome-specific, others are more broadly distributed $[9,10]$.

Repetitive sequences have a large influence on genome structure, function and evolution but, at the same time, complicate genomic analysis. These highly variable genome components, especially TEs, are subject of dynamic evolution mainly due to insertions, illegitimate and unequal recombination, and interchromosomal and tandem duplications [11]. In bread (hexaploid) wheat, polyploidization and the prevalence of TEs has resulted in massive gene duplication and movement. From a practical point of view, repetitive sequences constitute a potential source of a wide range of markers useful for genome diversity and evolution analysis, genetic mapping and marker-assisted selection. Among them we can find markers based on short tandem repeats, such as Sequence Tagged Microsatellite Sites (STMS) [12] and Simple Sequence Repeats (SSRs) (reviewed in [13]), or markers based on transposable elements like: sequence-specific amplification polymorphism (SSAP) [14], retrotransposon based insertion polymorphism (RBIP) [15], interretrotransposon amplified polymorphism (IRAP) and retrotransposon-microsatellite amplified polymorphism (REMAP) [16], repeat junction- junction marker (RJJM) [17], insertion-site-based polymorphism (ISBP) [18,19], and repeat junction marker (RJM) [20].

The complete characterization of TEs, as well as the elucidation of their distribution across genomes and the mechanisms responsible for that distribution, constitutes essential information for understanding the nature and consequences of genome size variations between different species, as well as the large-scale organization and evolution of plant genomes. However, this type of analysis is hindered by the large genome and the polyploid nature of bread wheat. The International Wheat Genome Sequencing Consortium (IWGSC, [21] has adopted the flow-sorted chromosome arms genomic approach to the analysis of the wheat genome, achieving a great reduction in complexity [22]. The combination of second generation sequencing technologies and DNA from flow-sorted chromosomes and chromosome arms became base for survey sequencing of all chromosome arms of wheat [3]. With some limitations in the building of contigs/scaffolds, this survey sequences provides unique opportunity to study the repetitive portion of each chromosome individually, enables comparisons among different chromosomes [23], and may enable identification of chromosome or genome specific sequences.

As members of IWGSC [21], our laboratory obtained a survey sequence of wheat chromosome arms 4DS and 4DL and, through a combination of different approaches, a virtual map including 1973 syntenic genes was built and $\sim 5,700$ genes were predicted on bread wheat chromosome 4D [24]. An even distribution of repetitive elements was also reported in both arms [24], but the repeat fraction of this chromosome was not characterized. Here, we focused on chromosome 4D repeatome and analyzed and characterized the repetitive sequences of chromosome 4D arms obtained through Roche 454 sequencing technology (JROL0000000, [24] and compared it with the 4A, 4B and $4 \mathrm{D}$ sequences obtained through Illumina sequencing technology [3], hereinafter differenced with the subscripts 454 and I, respectively. Identified transposable elements were analyzed and sorted by class and classified to families. Novel LTR subfamilies were identified, analyzed, and characterized using specific bioinformatics tools. Their physical localization and distribution along the whole wheat genome was assessed by fluorescent in situ hybridization (FISH).

\section{Results and discussion}

\section{Quantification of repetitive sequences from wheat} homeologous group 4 chromosome arms

The repetitive elements were assessed through homologybased comparison with the MIPS Repeat Element Database using the assemblies obtained from Roche 454 
survey sequences of chromosome arms of wheat chromosome $4 \mathrm{D}\left(4 \mathrm{DS}_{454}\right.$ and $\left.4 \mathrm{DL}_{454}\right)$ and Illumina sequences of all chromosome arms of wheat chromosome group 4 . Computational identification, classification and masking of repetitive elements, including low complexity regions using the RM software yielded $67.4 \%$ and $65.6 \%$ for $4 \mathrm{DS}_{454}$ and $4 \mathrm{DL}_{454}$ and $55.0 \%$ and $38.7 \%$ for $4 \mathrm{DS}_{\mathrm{I}}$ and $4 \mathrm{DL}_{\mathrm{I}}$ masked bases, respectively (Table 1 ; Additional file 1: Tables S1, Additional file 2: Table S2 and Additional file 3: Table S3). On the other hand, $4 \mathrm{AS}_{\mathrm{I}}$, $4 \mathrm{AL}_{\mathrm{I}}, 4 \mathrm{BS}_{\mathrm{I}}$ and $4 \mathrm{BL}_{\mathrm{I}}$ were composed of $63.8 \%, 56.6 \%$, $59.8 \%$ and $57.0 \%$ of repetitive sequences, respectively (Table 1; Additional file 1: Tables S1, Additional file 2: Table S2 and Additional file 3: Table S3). Similarly, repetitive DNA contents of the diploid $\mathrm{A}$ and $\mathrm{D}$ genome contributors of hexaploid wheat, T. urartu and Ae. tauschii, were reported to be $67 \%$ [25] and $66 \%$ [26], respectively.

Comparisons among the percentage of the repeat fraction of chromosome arms reported using Roche 454 sequencing technology [19,27-31] and the ones obtained through Illumina [21], suggest that the first ones constitute a better representation on the real status (Table 2). This assumption is further supported by previous estimations of $75-90 \%$ repetitive sequence content of bread wheat genome $[19,20]$. It has been reported that the longer Roche 454 reads as compared to the Illumina reads, results in an improvement in mapping of repetitive regions [32]. In addition to the sequencing platform, it is necessary to take into account that the estimation of repetitive DNA content depends on the method used. Using mathematically defined repeats (MDRs) [33] it was estimated that the assembly of all chromosome survey sequences of wheat contains $76.6 \%$ of MDRs - 20mers with abundance over 1000 copies [3]. The difference observed could be caused by the limited representation in the databases used for masking.

The repetitive regions of $4 \mathrm{DS}_{454}$ and $4 \mathrm{DL}_{454}$ were almost homogeneously distributed along both chromosome arms [24] what, likely, may be due to limitations of repetitive sequences assembly, used genetic map and GenomeZipper which are positively biased toward the gene-containing regions [34].

\section{Classification of repetitive sequences from wheat homeologous group 4 chromosome arms}

The RM software was further used to classify recognizable repeat families. RNA retrotransposons were the most highly represented sequence repeats, accounting for $50 \%$ of all chromosome arms except for $4 \mathrm{DL}_{\mathrm{I}}$, in which it was estimated to be $33 \%$ of the total sequenced length (Table 1). However, retroelements represented between $74 \%$ to $92 \%$ of the total repeat fraction in the eight

Table 1 Repetitive elements identified in Triticum aestivum (var. Chinese Spring) homeologous group 4 chromosome arms

\begin{tabular}{|c|c|c|c|c|c|c|c|c|c|c|c|c|}
\hline & \multicolumn{3}{|l|}{$4 \mathrm{AS}_{\mathrm{I}}$} & \multicolumn{3}{|l|}{ 4BS } & \multicolumn{3}{|l|}{ 4DS } & \multicolumn{3}{|l|}{$4 \mathrm{DS}_{454}$} \\
\hline & $\#$ & Length & $\%$ & $\#$ & Length & $\%$ & $\#$ & Length & $\%$ & $\#$ & Length & $\%$ \\
\hline Retroelements & 315566 & 167160901 & 59.21 & 268357 & 164853117 & 53.49 & 112608 & 69191801 & 48.69 & 20607 & 19344019 & 50.12 \\
\hline DNA transposons & 38628 & 11506157 & 4.08 & 49240 & 15027055 & 4.88 & 25899 & 8060073 & 5.67 & 9835 & 5969965 & 15.47 \\
\hline Unclassified: & 1117 & 194275 & 0.07 & 1303 & 311827 & 0.1 & 707 & 161819 & 0.11 & 1849 & 539049 & 1.40 \\
\hline Small RNA: & 265 & 47072 & 0.02 & 319 & 51039 & 0.02 & 184 & 37472 & 0.03 & 69 & 22419 & 0.06 \\
\hline Satellites: & 819 & 144631 & 0.05 & 3929 & 2914989 & 0.95 & 569 & 116493 & 0.08 & 21 & 2468 & 0.01 \\
\hline Simple repeats: & 10379 & 583006 & 0.21 & 12326 & 709023 & 0.23 & 5394 & 329739 & 0.23 & 1106 & 47817 & 0.12 \\
\hline Low complexity: & 9628 & 497086 & 0.18 & 9794 & 510460 & 0.17 & 5487 & 286086 & 0.2 & 1293 & 83621 & 0.22 \\
\hline \multirow[t]{3}{*}{ Total } & 376402 & 180133128 & 63.82 & 345268 & 184377510 & 59.84 & 150848 & 78183483 & 55.01 & 34780 & 26009358 & 67.4 \\
\hline & \multicolumn{3}{|l|}{$4 A L_{1}$} & \multicolumn{3}{|l|}{$4 \mathrm{BL}_{\mathrm{I}}$} & \multicolumn{3}{|l|}{$4 \mathrm{DL}_{\mathrm{I}}$} & \multicolumn{3}{|l|}{$4 \mathrm{DL}_{454}$} \\
\hline & $\#$ & Length & $\%$ & $\#$ & Length & $\%$ & $\#$ & Length & $\%$ & $\#$ & Length & $\%$ \\
\hline Retroelements & 343182 & 180465664 & 49.86 & 269612 & 124807259 & 50.19 & 328691 & 116699122 & 33.57 & 15107 & 14212381 & 52.61 \\
\hline DNA transposons & 71092 & 21753200 & 6.01 & 51423 & 14335731 & 5.77 & 53046 & 12099355 & 3.48 & 6296 & 3030966 & 11.22 \\
\hline Unclassified: & 2334 & 561199 & 0.16 & 1405 & 345580 & 0.14 & 1622 & 328062 & 0.09 & 1258 & 342679 & 1.27 \\
\hline Small RNA: & 535 & 101456 & 0.03 & 273 & 42903 & 0.02 & 349 & 46900 & 0.01 & 55 & 14356 & 0.05 \\
\hline Satellites: & 1695 & 299708 & 0.08 & 3051 & 1041847 & 0.42 & 2128 & 296676 & 0.09 & 16 & 2622 & 0.01 \\
\hline Simple repeats: & 13225 & 727067 & 0.2 & 10237 & 571582 & 0.23 & 47444 & 3205296 & 0.92 & 750 & 31119 & 0.12 \\
\hline Low complexity: & 14467 & 772219 & 0.21 & 8455 & 453240 & 0.18 & 35801 & 1723366 & 0.5 & 1104 & 74281 & 0.27 \\
\hline Total & 446530 & 204680513 & 56.55 & 344456 & 141598142 & 56.95 & 469081 & 134398777 & 38.66 & 24586 & 17708404 & 65.55 \\
\hline
\end{tabular}

The chromosome arms are expressed by a number that indicates the homeologous group followed by a letter that indicates the genome (A, B or D) and the chromosome arm (S: short; L: long). The subscripts refer to the technology used for sequencing (I: Illumina; 454: Roche 454). For each element class the number of elements (\#), the length of the sequence occupied by these elements (length) and the percentage of the sequence that is covered by repetitive elements (\%) are given. 
Table 2 Comparison of the repeat content of $T$. aestivum chromosomes and chromosome arms obtained through different sequencing technologies

\begin{tabular}{|c|c|c|c|c|}
\hline \multirow[t]{2}{*}{ Chromosome } & \multicolumn{2}{|l|}{454} & \multicolumn{2}{|l|}{ Illumina } \\
\hline & $\begin{array}{l}\text { Repeat } \\
\text { content }\end{array}$ & Coverage & $\begin{array}{l}\text { Repeat } \\
\text { content }\end{array}$ & Coverage \\
\hline $3 A S$ & $79 \%$ [28] & $3.2 \%$ & $62 \%$ & $56 \%$ \\
\hline $3 B$ & $86 \%[19]$ & & - & $64 \%$ \\
\hline $4 A S$ & $80 \%[29]$ & - & $63 \%$ & $89 \%$ \\
\hline $4 \mathrm{AL}$ & $73 \%$ [29] & - & $56 \%$ & $66 \%$ \\
\hline $4 B S$ & - & & $60 \%$ & $97 \%$ \\
\hline $4 B L$ & - & & $57 \%$ & $58 \%$ \\
\hline 4DS & $67 \%$ & $44 \%$ & $55 \%$ & $62 \%$ \\
\hline $4 \mathrm{DL}$ & $66 \%$ & $29 \%$ & $39 \%$ & $83 \%$ \\
\hline $5 A S$ & $76 \%[27]$ & $21 \%$ & $67 \%$ & $67 \%$ \\
\hline $5 A L$ & $82 \%[27]$ & & $64 \%$ & $60 \%$ \\
\hline $5 B S$ & $72 \%[31]$ & $6 \%$ & $60 \%$ & $60 \%$ \\
\hline $5 B L$ & $71 \%$ [31] & $8 \%$ & $55 \%$ & $72 \%$ \\
\hline $6 B S$ & $77 \%[30]$ & $55.6 \%$ & $58 \%$ & $51 \%$ \\
\hline $6 \mathrm{BL}$ & $86 \%$ [30] & $54.9 \%$ & $59 \%$ & $52 \%$ \\
\hline
\end{tabular}

The compared chromosomes and chromosome arms are listed on the first column. The following columns show the repeat content, expressed as the percentage of the total sequenced bases and the chromosome coverage calculated as the ratio between the available chromosome or chromosome arm length and the predicted one [74] using as input 454 or Illumina sequences. When data was obtained from literature, the references are cited.

datasets of the homeologous group 4 chromosome arms. The retroelements were followed in abundance by DNA elements, which comprised between $7 \%$ to $23 \%$ of total repeats. Thus, the DNA and RNA transposons represented more than $96 \%$ of the repeat fraction for all the chromosome arms. DNA transposons and retrotransposons were further subclassified, according to the Wicker's criteria [6], revealing that for 4D chromosome arms, CACTA/En-Spm DNA-transposons and Gypsy retrotransposons were the most abundant superfamilies (Figure 1).

The analysis and characterization of satellites, simple repeats and low complexity regions were performed along the wheat homeologous group 4 chromosome arms sequences included in this study (Table 1; Additional file 1: Table S1, Additional file 2: Table S2 and Additional file 3: Table S3). Small RNA, satellites, simple repeats and low complexity sequences represented only small proportions of assemblies of all chromosome arms (Table 1). This finding is not surprising, because these loci derive from repetitive AT and GC-rich sequences that may be collapsed or represented by uneven read coverage in Illumina sequences [35]. This assumption is corroborated by the finding that the GAA microsatellite is not observed within the most abundant microsatellites detected (Additional file 2: Table S2), although its presence has been previously evidenced by using FISH, where the GAA designed probe creates large

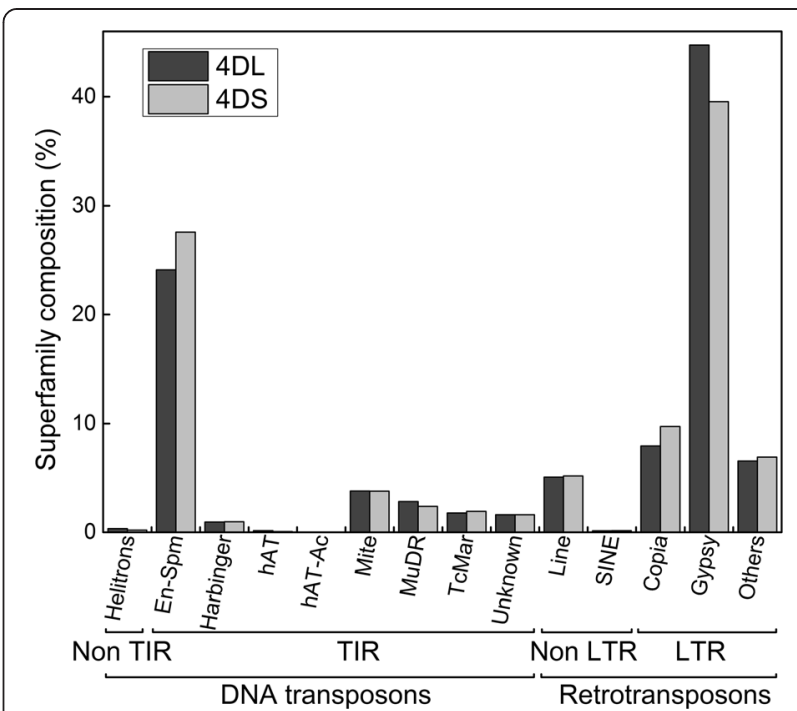

Figure 1 Distribution of DNA and RNA transposon superfamilies on 4D chromosome arms. Bars represent the percentage of each superfamily with respect to the total DNA or RNA transposable elements, for 4DL (dark grey bars) and 4DS (light grey bars). Classification was performed as suggested in [6].

blocks of signal on all chromosomes [36]. On the other hand several of the trinucleotide SSRs identified as the most abundant in the survey sequences provide on FISH weak disperse signals (Kubaláková, personal communication). The presence of SSRs was analyzed in order to search for new putative markers for physical and genetic mapping. The frequency of SSRs ranged from one SSR per 24 to $27 \mathrm{~kb}$ for $4 \mathrm{AS}_{\mathrm{I}}, 4 \mathrm{AL}_{\mathrm{I}}, 4 \mathrm{BS}_{\mathrm{I}}, 4 \mathrm{BL}_{\mathrm{I}}, 4 \mathrm{DS}_{\mathrm{I}}, 4 \mathrm{DS}_{454}$ and $4 \mathrm{DL}_{454}$ chromosome arms. For $4 \mathrm{DL}_{\mathrm{I}}$ assemblies, the frequency was notably higher reaching one SSR per $7.2 \mathrm{~kb}$. Variable SSR frequency has been reported among other chromosomes or chromosome arms. For example, the frequency reported for 5BS and 5BL was one SSR per 19 and $23 \mathrm{~kb}$, respectively, whereas $3 \mathrm{~B}$ and $3 \mathrm{AS}$ chromosomes have a SSR frequency of one SSR per 6.1 and $10.4 \mathrm{~kb}$, respectively [19,28]. The SSR frequencies are higher in transcribed regions than in non-coding regions, being the SSR frequency inversely related to the proportion of repetitive DNA [37]. Thus, the lower repeat content of $4 \mathrm{DL}_{\mathrm{I}}$ respect either to $4 \mathrm{DL}_{454}$ or to the other chromosome arms from the homeologous group 4 agrees with the higher frequency of SSR. This is also in agreement with the gene content calculated for chromosome 4D [24]. The analysis of SSR motifs according to the size of repeat units revealed that dinucleotides and trinucleotides were the most abundant SSRs (Figure 2). In comparison, the trinucleotide constitutes the most frequent SSR motif in Brachypodium, rice and maize whereas papaya shows a higher frequency of dinucleotide motifs and soybean a higher frequency of tetranucleotide motifs [38]. Microsatellites were further 


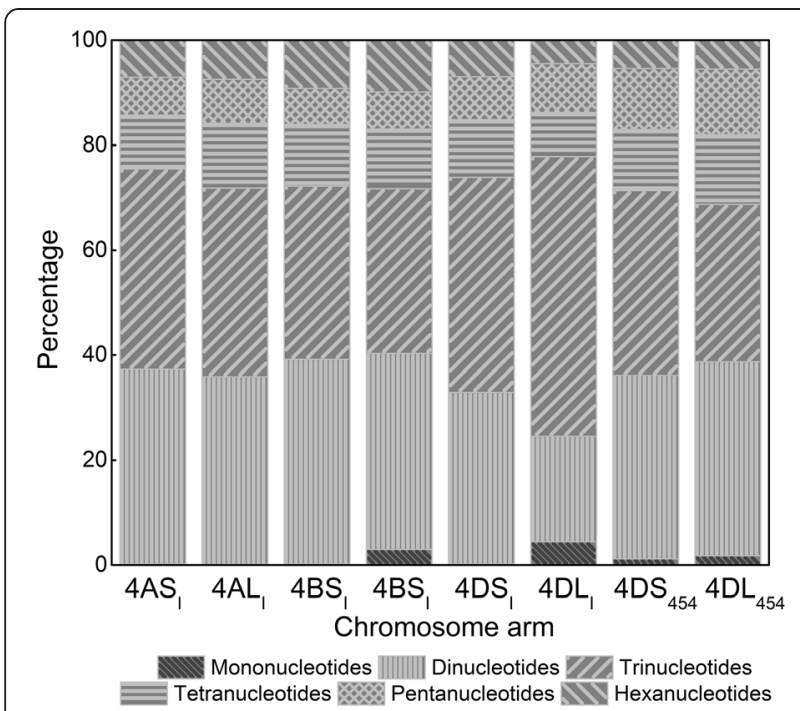

Figure 2 SSR classification according to the size of repeat units. The graph illustrates the frequency of the different sizes of the SSRS repeat units classified as dinucleotides, trinucleotides,

tetranucleotides, pentanucleotides and hexanucleotides across the homeologous group 4 chromosome arms from $T$. aestivum obtained through Illumina (4AS, 4AL $, 4 B S_{1}, 4 \mathrm{BL}_{1}, 4 \mathrm{DS}_{\mid}$and $\left.4 \mathrm{DL}_{\mid}\right)$and 454 $\left(4 \mathrm{DS}_{454}\right.$ and $\left.4 \mathrm{DL}_{454}\right)$ sequencing technologies.

divided into three classes, AT-rich (greater than $50 \%$ A or $\mathrm{T}$ in the motif), AT/GC-balanced (limited to di- and tetra-nucleotide motifs that fit this criterion) and GC-rich motifs. The analysis revealed the predominance of AT/GC balanced motifs on the chromosome arms $4 \mathrm{AS}_{\mathrm{I}}, 4 \mathrm{AL}_{\mathrm{I}}$, $4 \mathrm{BS}_{\mathrm{I}}, 4 \mathrm{BL}_{\mathrm{I}}$ and $4 \mathrm{DS}_{454}$ whereas the most abundant on $4 \mathrm{DL}_{454}, 4 \mathrm{DS}_{\mathrm{I}}$ and $4 \mathrm{DL}_{\mathrm{I}}$ were the GC-rich motifs (Table 3 ). The combination of both analysis, i.e., the size and nucleotide composition of the repeat unit, revealed that more than the $80 \%$ of the dinucleotides belongs to the AT/GC-balanced class, mainly (GA)n/(TC)n, which is also the most abundant dinucleotide motif in Brachypodium and Arabidopsis while in papaya and soybean the most abundant dinucleotide is AT/TA [38]. Except for $4 \mathrm{DL}_{454}$, the $\mathrm{GC}$-rich are the prevalent trinucleotide motif in all chromosome arms as it was described for Brachypodium and rice whereas in maize, wheat, papaya, Arabidopsis and soybean AT-rich

Table 3 SSR classification according to the classes

\begin{tabular}{lllllllll}
\hline & $\mathbf{4 A S}_{\mathbf{I}}$ & $\mathbf{4 B S}_{\mathbf{I}}$ & $\mathbf{4 D S}_{\mathbf{|}}$ & $\mathbf{4 D S}_{\mathbf{4 5 4}}$ & $\mathbf{4 A L}_{\mathbf{|}}$ & $\mathbf{4 B L}_{\mathbf{|}}$ & $\mathbf{4 D L}_{\mathbf{|}}$ & $\mathbf{4 D L}_{\mathbf{4 5 4}}$ \\
\hline AT-rich & 32,4 & 25,4 & 25,7 & 36,4 & 28,7 & 23,9 & 10,2 & 23,0 \\
GC-rich & 29,0 & 28,5 & 37,4 & 30,1 & 29,3 & 29,8 & 66,8 & 40,4 \\
$\begin{array}{l}\text { AT/GC } \\
\text { balanced }\end{array}$ & 38,5 & 46,1 & 36,8 & 33,5 & 41,9 & 46,3 & 23,0 & 36,6
\end{tabular}

Microsatellites were classified into three classes, AT-rich: greater than $50 \%$ A or $\mathrm{T}$ in the motif; GC-rich: greater than $50 \% \mathrm{G}$ or C in the motif; AT/GC-balanced: equal amount of GC and AT, along the six chromosome arms obtained through Illumina and the two obtained through 454 sequencing technologies. trinucleotide repeats were the majority [38]. Interestingly, trinucleotides motifs represent $\sim 50 \%$ of the $4 \mathrm{DL}_{\mathrm{I}} \mathrm{SSRs}$ mainly composed of CCG/CGG motifs ( 75\%). Finally, the AT-rich tetranucleotide SSRs prevail in the majority of the group 4 homeologous chromosome arm survey sequences. These vast data were used to identify SSR motifs specific for the tested chromosome arms or whole chromosomes and we tested this information by their physical localization on metaphase chromosomes. Identification of SSRs with chromosome distinct pattern may have practical implications.

The SSR markers still have potential for whole genome or sub-genome mapping [39,40] and breeding [13]. Additionally, some of the SSRs were found very useful as physical markers for cytogenetic mapping, metaphase chromosome identification [41] and enhancing chromosome sorting by FISHIS [42]. Since most of the di and tri-nucleotide SSRs were already localized [43] we focused on SSRs with longer subunit. The comparison of sequence occurrence of unique SSR motifs among chromosomes and chromosome arms allowed identification of SSRs suggestive to be putative arm-specific (Additional file 2: Table S2). The (CAGCG)n/(CGCTG)n and (CCGTA)n/(TACGG)n motifs showed specificity for 4DL and (CGTAG)n/(CTACG)n showed specificity for 4BL. Additionally, (TTACG)n/(CGTAA)n was found specific for chromosome 4D. FISH localization on metaphase chromosomes showed that microsatellites produced weak dispersed signals on almost all chromosomes (data not shown). These findings suggest that quantitative assessment of SSRs in the survey sequence assembly may not be representative due to, already above discussed, the possibility of collapsing of highly repetitive tandem repeats in assemblies of short sequencing reads, but catalog of available microsatellites and other repeats can provide useful information for marker candidate sequence identification and marker development.

\section{Identification and annotation of novel LTR retrotransposons}

LTR retrotransposons account for a significant fraction of many genomes and even are the predominant component of some large genomes [6]. Typical structural characteristics include: 1) two highly similar LTR sequences; 2) target site duplications; 3) a primer binding site and a polypurine tract; 4) protein-coding domains for enzymes important to retrotransposition [6]. Additionally, non-autonomous LTR retrotransposons have been described in plants as large retrotransposon derivatives (LARDs) and terminal repeat retrotransposons in miniature (TRIMs), both of which have the typical features of LTR retrotransposons but lack protein-coding capability in their internal domain $[44,45]$. 
The whole 4DS and 4DL scaffolds were further scanned for LTR retrotransposons using the bioinformatics tools LTR_FINDER [46] and LTR_STRUC [47]. The mentioned subset of data was chosen for novel LTR identification due to the larger size when compared to the Illumina contigs, as revealed by size frequency histograms (Additional file 4: Figure S1). The LTR_FINDER and LTR_STRUC outputs lead to 234 candidate sequences (Figure 3a), that were clustered using the CD-HIT interface [48], resulting in 214 unique LTR retrotransposon candidates. After manually search for previously defined elements against MIPS database following the criteria of [6], 171 putative retrotransposons were excluded (Figure 3a). The remaining 43 candidate elements were analyzed for the presence of LTR retrotransposon features using BLASTX searches at NCBI and GyDB [49], reducing the number of candidates for newly identified retrotransposons to six (Table 4). The BLASTX analysis also revealed that likely complete transposon-related proteins were present in four out of the six candidates (JROL01007197, JROL01007734, JROL01000922 and JROL01008273), as judged by the coverage of the alignments with reported proteins, whereas the other two, JROL01006440 and JROL01007833, showed small protein fragments and thus non-coding capacity. The fact that two out of four retrotransposon protein coding regions lack stop codons whereas the other two showed only one indicate that such candidates could encode functional protein sequences. Notice that the presence of few stop codons may not directly imply the absence of functionality of a TE family since only recently inserted elements have not been subject to mutations and could be taken as functional. The identity and coverage of the

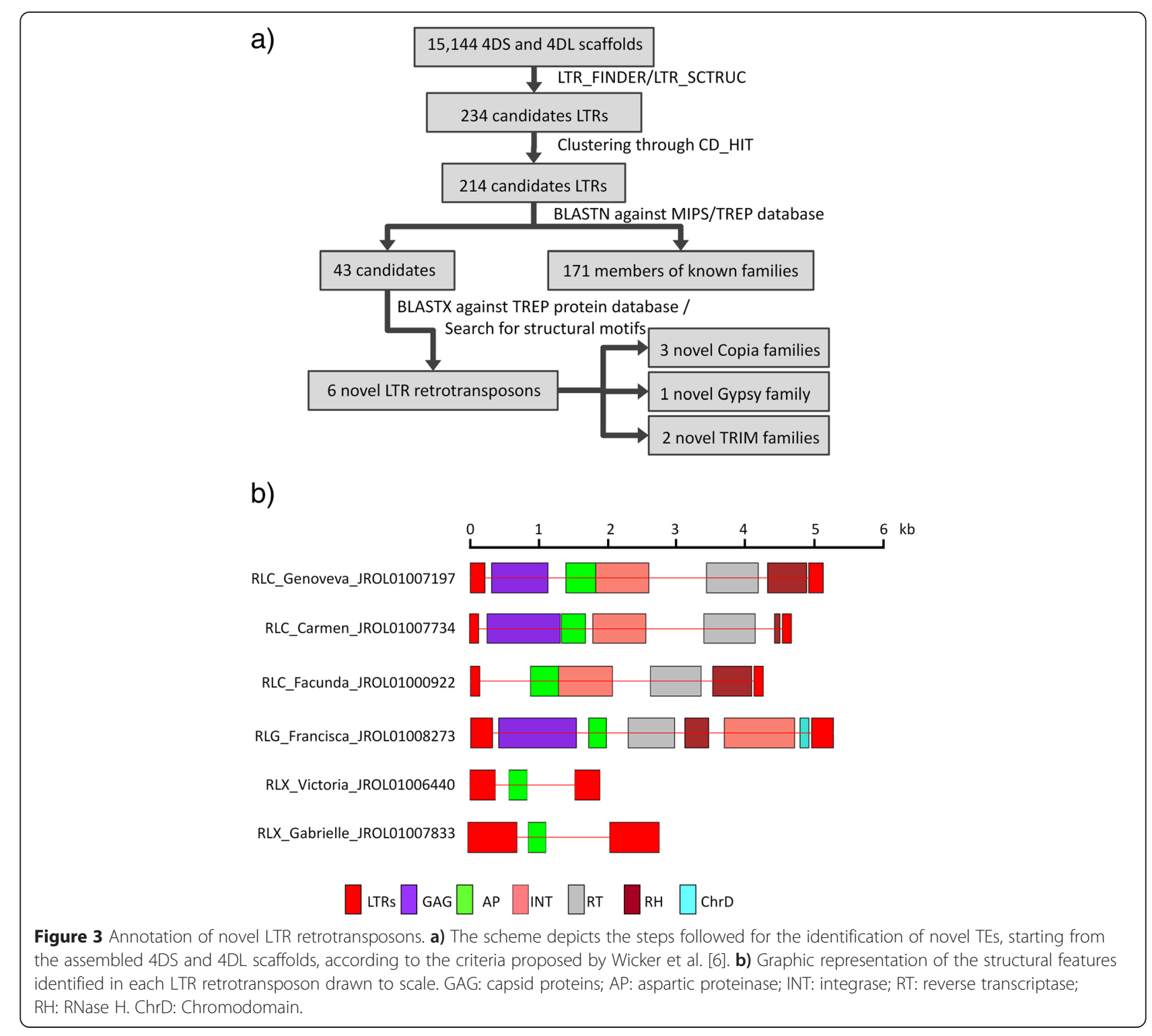


Table 4 Description of the 6 LTR retrotransposon candidates identified on 4D chromosome scaffolds

\begin{tabular}{|c|c|c|c|c|c|c|c|c|c|c|}
\hline LTR retrotranspoon & program & LTR retrotransposonsize & $\begin{array}{l}\# \text { in } \\
\text { genome }\end{array}$ & $\begin{array}{l}\text { LTR region } \\
\text { similarity }\end{array}$ & 5'-LTR size & 3'-LTR size & $\begin{array}{l}\text { Insertion time } \\
\text { (years } \times 10^{6} \text { ) }\end{array}$ & TSD & PBS & PPT \\
\hline RLC_Genoveva_JROL01007197 & STRUC/FINDER & 5132 & 30 & 0.978 & 215 & 215 & 0.70 & GAGGC & Lys_TT & GCCTCCCTCTTCCTC \\
\hline RLC_Carmen_JROL01007734 & FINDER & 4597 & 40 & 0.977 & 131 & 131 & 0.27 & - & SerTGA & ССАТСТTСТTССТСС \\
\hline RLC_Facunda_JROL01000922 & FINDER & 4842 & 757 & 0.942 & 138 & 139 & 4.65 & - & MetCAT & GATACTGCGGGGGGA \\
\hline RLG_Francisca_JROL01008273 & STRUC/FINDER & 5279 & 21 & 0.969 & 321 & 321 & 0.70 & CTGTC & SerGCT & TCTCCTGGTCCTCCC \\
\hline RLX_Victoria_JROL01006440 & FINDER & 1898 & 121 & 0.960 & 375 & 375 & 0.97 & - & MetCAT & TCATCCTCTCGCCCT \\
\hline RLX_Gabrielle_JROL01007833 & STRUC & 2644 & 33 & 0.935 & 685 & 680 & 6.11 & ACATT & MetCAT & $\begin{array}{l}\text { ATAGCTTCGTTCCAAG } \\
\text { AAGGAGGGGA }\end{array}$ \\
\hline
\end{tabular}

The designations on the new LTR retrotransposons are indicated in column 1. The number of genomic repetitions for each candidate LTR retrotransposon was estimated by searching against the T. aestivum

chromosome arm contigs deposited in the URGI database (\# in genome). LTR: Long terminal repeat; TSD: target site duplication; PBS: primer binding site; PPT: polypurine tract. The last column indicates the presence $(+)$ or absence $(-)$ of retrotransposon proteins when BLAST searched against the TREP protein database. 
alignments demonstrate that the novel LTR retrotransposons are members of known superfamilies but constitute novel LTR retrotransposon families (Table 5). Their classification was carried out following the current proposed system [6], revealing that three of the newly identified LTR retrotransposons belonged to the Copia superfamily, one was Gypsy and the other two were non autonomous terminal repeat retrotransposons in miniature (TRIMs) and thus designations were assigned to the six new families (Tables 5 and 6, Figure 3b). The insertion time of the six newly identified LTR retrotransposons was estimated based on the assumption that the sequences of the two LTRs were identical at the time of integration and accumulated point mutations independently with time. Thus, the nucleotide substitution rate between the two LTRs, considered to reflect the time elapsed since the insertion event, was estimated to be in the range of $0.2710^{6}$ to $6.1110^{6}$ years (Table 4 ).

\section{Identification of members of the novel LTR retrotransposon families}

The presence of full-length copies of the novel LTR retrotransposon in genome was tested, using the candidate LTR retrotransposons as probes against the $T$. aestivum chromosome arm assemblies acquired from the IWGSC database, following the criteria proposed by [6]. There were identified 21 to 757 copies for each candidate, being RLC_Facunda_JROL01000922-1 the most abundant one (Table 4).

However, such values are probably miscalculated due to the short length of the sequences deposited in the databases; thus, a single unique large LTR retrotransposon could give rise to several hits. To address this, we adopted an additional approach consisting of BLASTN searches against the $T$. aestivum (WGS project accession CALP000000000; [50]) and Ae. tauschii (WGS project accession AOCO000000000; [51]) whole genome shotgun sequence (wgs) databases using the six candidate LTR retrotransposons as probes. The resulting sequences were used as input for the LTR_FINDER and LTR_STRUC programs and the output sequences were extracted from the wgs and manually analyzed to verify the identity with the probed LTR retrotransposon. Such procedure allowed identification of one member of the LTR retrotransposon family for candidates RLC_Genoveva_JROL01007197-1 and RLX_Gabrielle_JROL01007833-1, two for RLC_Facunda JROL01000922-1 and RLG_Francisca_JROL01008273-1 and three for RLC_Carmen_JROL01007734-1 (Additional file 5: Table S4). Interestingly, thirty one new LTR retrotransposons were identified when probed with RLX_Victoria_JROL01006440-1 (Additional file 5: Table S4).

Finally, all the positive hits obtained through BLASTN search of T. aestivum and Ae. tauschii wgs probed with the six candidate LTR retrotransposons were additionally BLAST searched against a local database, constructed by adding to the MIPs database the six novel LTR retrotransposons. The alignments among the wgs and the local database were manually analyzed. To be considered a candidate LTR retrotransposon copy the wgs needed to: i) show identity to the candidate exclusively or, b) exhibit remarkably higher identity to the probed LTR retrotransposon than to any other LTR retrotransposon. Sequences that fulfilled these parameters were extracted from wgs. This approach showed that at least one

Table 5 BLASTX alignment of coding sequences encoded by the novel LTR retrotransposons with TREP database

\begin{tabular}{|c|c|c|c|c|c|c|}
\hline LTR retrotransposon & $\begin{array}{l}\text { TREP protein } \\
\text { code }^{1}\end{array}$ & $\begin{array}{l}\text { LTR retrotransposon } \\
\text { associated }^{2}\end{array}$ & Score $^{3}$ (bits) & Identity $^{4}$ & $\begin{array}{l}\text { Conservative } \\
\text { substitutions }^{5}\end{array}$ & Coverage $^{6}$ \\
\hline \multirow[t]{2}{*}{ RLC_Genoveva_JROL01007197 } & PTREP238 & TREP3154 & 1130 & $64 \%$ & $78 \%$ & $84 \%$ \\
\hline & (1515 aа) & Copia, RLC_Olivia_42j2-1 & & & & \\
\hline \multirow[t]{2}{*}{ RLC_Carmen_JROL01007734 } & PTREP238 & TREP3154 & 879 & $54 \%$ & $67 \%$ & $75 \%$ \\
\hline & (1515 aа) & Copia, RLC_Olivia_42j2-1 & & & & \\
\hline \multirow[t]{2}{*}{ RLC_Facunda_JROL01000922 } & PTREP120 & TREP2012 & 1154 & $58 \%$ & $74 \%$ & $85 \%$ \\
\hline & (1121 аa) & Copia, RLC_Zenia_AY853252-1 & & & & \\
\hline \multirow[t]{2}{*}{ RLG_Francisca_JROL01008273 } & PTREP249 & TREP3203 & 387 & $32 \%$ & $49 \%$ & $93 \%$ \\
\hline & (1536 aа) & Gypsy, RLG_Latidu_10k23-1 & & & & \\
\hline \multirow[t]{2}{*}{ RLX_Victoria_JROL01006440 } & PTREP64 & TREP99 & 130 & $37 \%$ & $58 \%$ & $20 \%$ \\
\hline & (1717 aа) & Gypsy,RLG_Cereba_AY040832-2 & & & & \\
\hline \multirow[t]{2}{*}{ RLX_Gabrielle_JROL01007833 } & PTREP63 & TREP98 & 104 & $32 \%$ & $51 \%$ & $22 \%$ \\
\hline & (1520 aa) & Gypsy, RLG_Cereba_AY040832-1 & & & & \\
\hline
\end{tabular}

${ }^{1}$ code of the protein that showed the highest identity to the scaffold. Its length is indicated in parenthesis; ${ }^{2}$ the code and name of the retrotransposon associated with the mentioned proteins; ${ }^{3}$ maximal score of the alignments expressed in bits; ${ }^{4}$ percentage of identity of the alignments; ${ }^{5}$ percentage of conservative substitutions, i.e., the aligned amino acids are not identical but both side chains have similar biochemical properties. ${ }^{6}$ percentage of the protein sequences that aligned with the scaffold sequence. 
Table 6 Description of the novel LTR retrotransposons taxonomy and family members

\begin{tabular}{|c|c|c|c|c|c|c|}
\hline $\begin{array}{l}\text { Sequence } \\
\text { accession }\end{array}$ & JROL01007197 & JROL01007734 & JROL01000922 & JROL01008273 & JROL01006440 & JROL01007833 \\
\hline Family & Genoveva & Carmen & Facunda & Francisca & Victoria & Gabrielle \\
\hline Superfamily & Copia & Copia & Copia & Gypsy & & \\
\hline Class & Retrotransposon & Retrotransposon & Retrotransposon & Retrotransposon & Retrotransposon & Retrotransposon \\
\hline Order & LTR retrotransposon & LTR retrotransposon & LTR retrotransposon & LTR retrotransposon & LTR retrotransposon & LTR retrotransposon \\
\hline Insertion & RLC_Genoveva_JROL01007197 & RLC_Carmen_JROL01007734 & RLC_Facunda_JROL01000922 & RLG_Francisca_JROL01008273 & RLX_Victoria_JROL01006440 & RLX_Gabrielle_JROL01007833 \\
\hline $\begin{array}{l}\text { Structural } \\
\text { description }\end{array}$ & Autonomous retrotransposon & $\begin{array}{l}\text { Autonomous } \\
\text { retrotransposon }\end{array}$ & $\begin{array}{l}\text { Autonomous } \\
\text { retrotransposon }\end{array}$ & Autonomous retrotransposon & $\begin{array}{l}\text { Non autonomous } \\
\text { retrotransposon (TRIM) }\end{array}$ & $\begin{array}{l}\text { Non autonomous } \\
\text { retrotransposon (TRIM) }\end{array}$ \\
\hline $\begin{array}{l}\text { Others } \\
\text { members }\end{array}$ & $\begin{array}{l}\text { See Additional file 5: } \\
\text { Tables S4 and } \\
\text { Additional file 6: } \\
\text { Table S5 }\end{array}$ & $\begin{array}{l}\text { See Additional file 5: } \\
\text { Tables S4 and } \\
\text { Additional file 6: } \\
\text { Table S5 }\end{array}$ & $\begin{array}{l}\text { See Additional file 5: } \\
\text { Tables \$4 and } \\
\text { Additional file 6: } \\
\text { Table S5 }\end{array}$ & $\begin{array}{l}\text { See Additional file 5: } \\
\text { Tables } \$ 4 \text { and } \\
\text { Additional file 6: } \\
\text { Table S5 }\end{array}$ & $\begin{array}{l}\text { See Additional file 5: } \\
\text { Tables S4 and } \\
\text { Additional file 6: } \\
\text { Table S5 }\end{array}$ & $\begin{array}{l}\text { See Additional file 5: } \\
\text { Tables S4 and } \\
\text { Additional file 6: } \\
\text { Table S5 }\end{array}$ \\
\hline
\end{tabular}


strong-hit copy was present in the wgs database for each of the six candidates, together with several partial copies (Table 6; Additional file 6: Table S5).

To elucidate the phylogenetic relationship among the members of each family, the six LTR candidates identified in the 4DS and 4DL scaffolds were individually aligned with the members of the respective families identified in wgs by the use of LTR_FINDER and LTR_STRUC and included some of the LTRs identified though BLASTN. For each candidate, the alignments included as outlayers LTRs that, in spite of being members of other families, were close to the new LTR candidates. The phylogenetic trees confirmed the existence of six new LTR retrotransposon families (Figure 4). For RLC_Carmen_JROL01007734-1 and RLC_Facunda_JROL01000922-1 there were included in the alignments the longer retrotransposons identified.

Special attention was centered in the LTR family RLX_Victoria_JROL01006440-1 since most members were identified by LTR_FINDER and/or LTR_STRUC and thus several structural information about them is available. Most of the members of the family ranged in size from 1898 to $3250 \mathrm{bp}$ and carried LTRs of 120 to $1051 \mathrm{bp}$, whereas one member was 8698 bp in length. Detailed insight in such member revealed that it was not a single retrotransposon but four Victoria LTR retrotransposons in tandem. Complete elements were flanked by 4 to 6-bp target site duplications. BLASTX alignment of the members of the family with retrotransposon proteins across the GyDb and NCBI databases revealed the presence of short fragments of some proteins, such as AP and INT. Since no complete ORF could be identified, it could be deduced that the internal domains of the elements lack coding capability. Regarding the internal region, the primer binding site was complementary to the methionine tRNA in $50 \%$ of the sequences, whereas $32 \%$ corresponded to other tRNAs and it could not be identified for $18 \%$ of the tRNAs. A 15-nt polypurine tract was identified upstream of the 3'LTR. As demonstrated through BLASTX searches in the NCBI and GyDB databases, none of the identified members of the

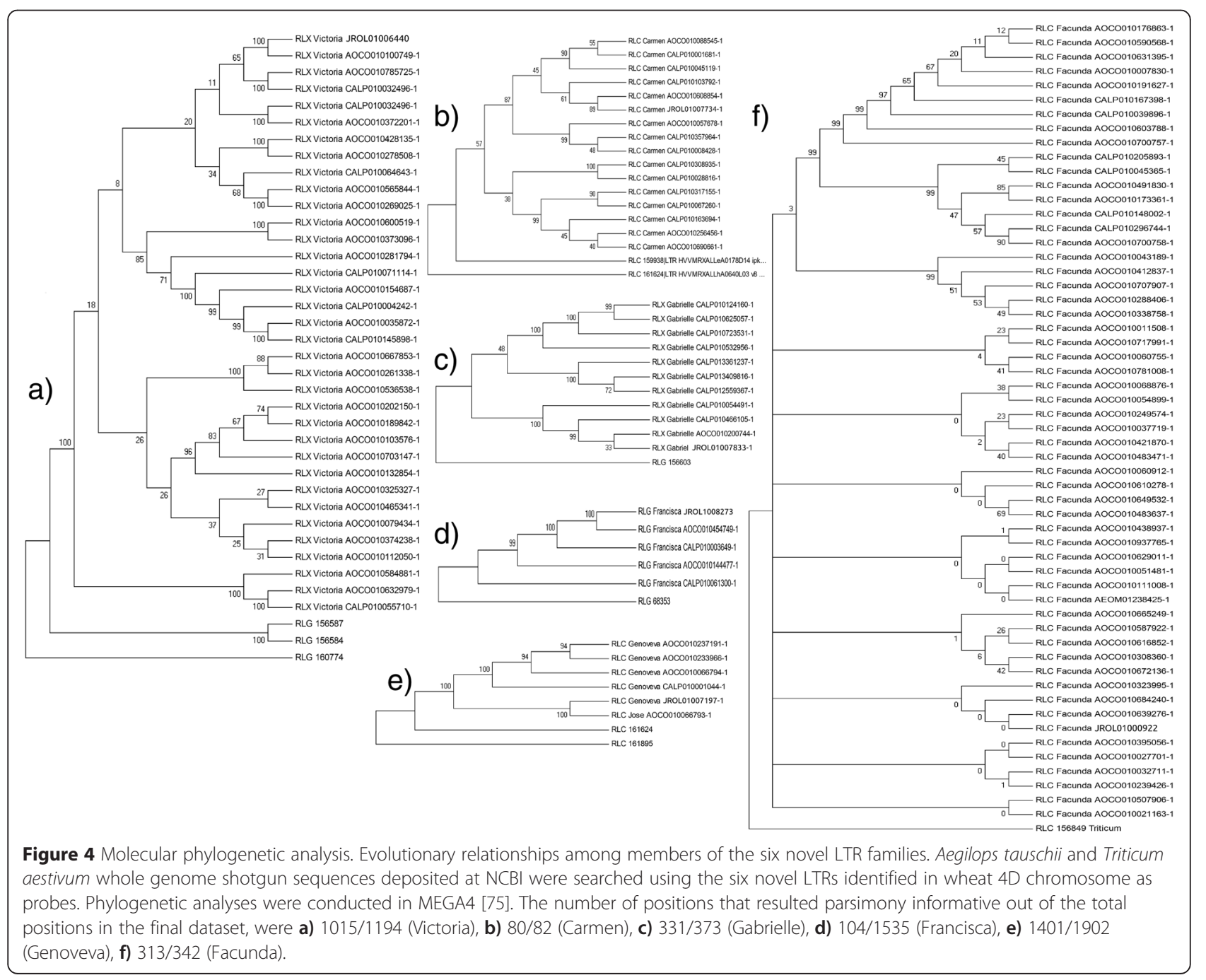


family possess the complete ORFs necessary to be considered an autonomous TE. Thus, taking into account the size of the members, the family was classified as TRIM non-autonomous LTR retrotransposons. The six novel retrotransposon families will be included in the next update of the Plant Genome and Systems Biology Repeat Element Database (PGSB-REdat).

The transposon insertion site based markers are specific and highly abundant, especially in large genomes where repetitive sequences represent major portions of genomic sequence, and became popular in plant genetic, physical mapping and diversity assessments. Several approaches were developed to visualize polymorphisms in the insertion sites and the most widely used in wheat are the RJM [20] or ISBP markers [18,19]. In light of this, identification and characterization of any new transposon adds to the pool of possible markers. After identification of six new LTR retrotransposons (Table 6) their DNA was amplified and labeled with fluorescent dye.
The resulting probes were hybridized on metaphase chromosomes. Most of the probes (Additional file 7: Table S6) provided weak and mostly randomly distributed unreliable signals on several chromosomes (similar to Figure 5a, data not shown). An exception was probe from LTR retrotransposon Carmen which provided signal in centromeric region of all chromosomes (Figure 5b) with highly varying intensity. Unfortunately, FISH analysis could not provide quantitative data which limits assessment of abundance of the retrotransposon for centromeres of particular chromosomes. Surprisingly, chromosome 4D showed very weak signal for this probe in all metaphase figures analyzed (Figure $5 \mathrm{c}$ and d). These findings support previously identified facts that repetitive elements and particularly transposons can, besides their selfish multiplication, play also an important role in evolution of genomes in moderating gene expression and creating new genes by exon reshuffling [52] or are part of important genome structures
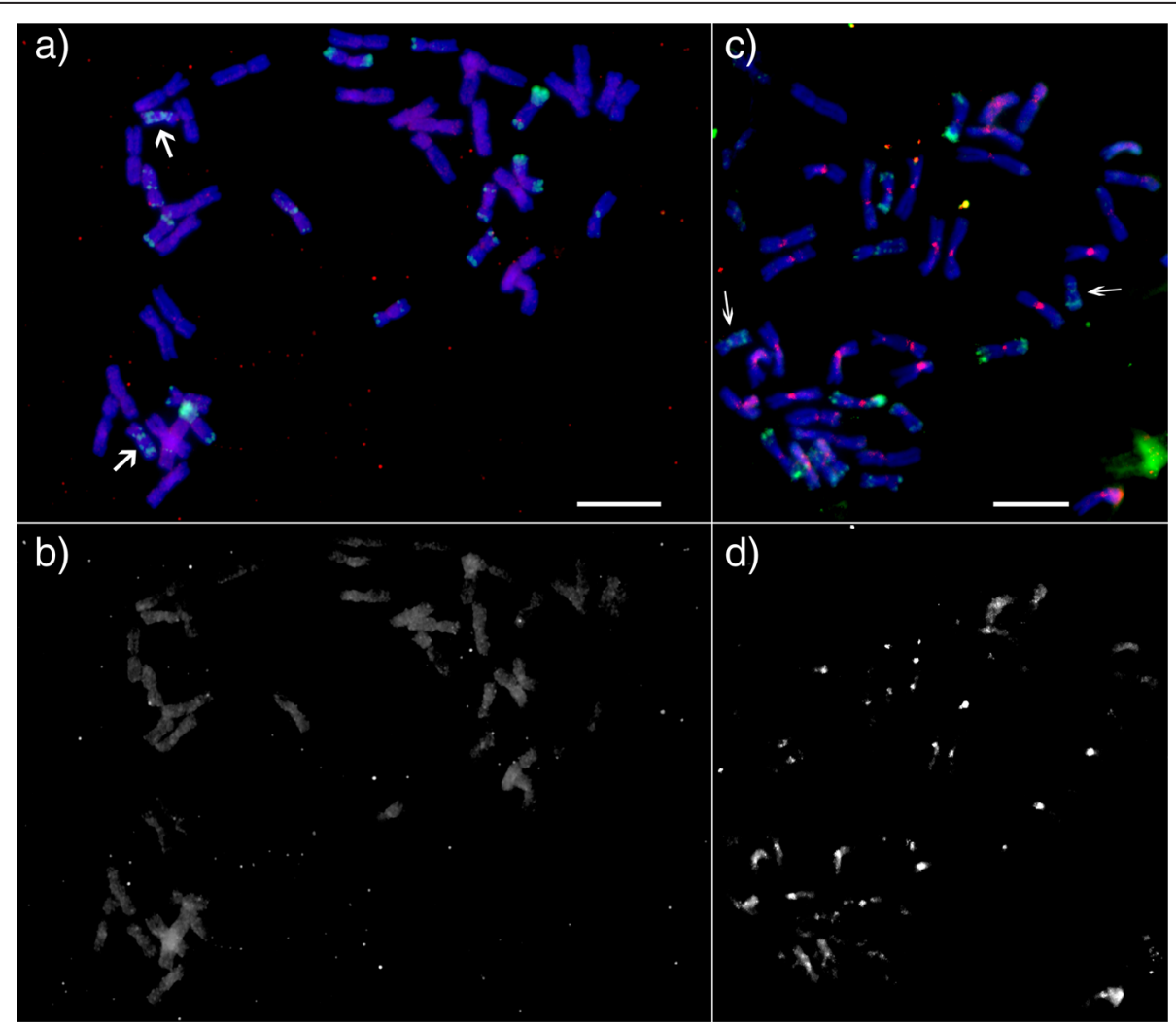

Figure 5 Physical localization of newly identified transposons. The identified retrotransposons were localized on metaphase spreads of vc. Chinese Spring using in situ hybridization with fluorescent labeled probes. Red color was used for the repetitive probes, Green color was used for Afa probe and Blue was stained chromosome DNA using DAPI. Arrows are pointing at 4D chromosome. a) Probes of all retrotransposons besides retrotransposon Carmen produced weak disperse signals on all chromosomes. The red signals represent distribution of retrotransposon Victoria. b) The red channel of figure A to demonstrate distribution of weak signals of Victoria probe on whole chromosomes. c) All probes derived from retrotransposon Carmen gave similar hybridisation pattern on metaphase chromosomes. Surprisingly, the retrotransposon was preferentially localized in centromeric region of all chromosomes with varying intensity. On few chromosomes were observed weak and dispersed signals on distal parts of chromosome arms too. d) The red channel of figure $\mathrm{C}$ to demonstrate distribution of weak signals of Carmen probe on whole chromosomes. Scale bars represent $10 \mu \mathrm{m}$. 
as centromeres and have ability to specifically target such structures $[53,54]$.

\section{Conclusion}

The present work constitutes the first insight of wheat homeologous group 4 chromosomes repetitive sequences analyzed at the chromosome arm level. Detailed study of repetitive elements becomes more interesting as it has been thought before, since repetitive elements seems to play important roles in genome structure and size variation and also contribute to the evolution of genes and their function. In accordance with results obtained for other grasses, CACTA/En-Spm and Gypsy were the most abundant DNA transposons and retrotransposons, respectively, suggestive of their conserved roles in genome regulation. The characterization of the tandem repeat content along the homeologous group 4 allowed creating a list of SSR motifs in wheat chromosomes of the homeologous group 4. Six novel LTR retrotransposon families were characterized, including three Copias, one Gypsy, and two TRIM LTR retrotransposons. In spite of the extensive research performed in Triticeae genomes and the high number of reported elements, the fact that six new elements could be identified indicates that new families probably remain to be described. However, for more detailed study of quantitative repeatome content and structure a reference sequence is crucial.

\section{Methods}

\section{Sequences from chromosome 4D}

Sequences from Triticum aestivum cv. Chinese Spring ditelosomic (DT) lines for the 4D chromosome arms were obtained through Roche 454 sequencing technology and assembled into 8141 and 7077 scaffolds for 4DS and $4 \mathrm{DL}$, respectively, hereafter named $4 \mathrm{DS}_{454}$ and $4 \mathrm{DL}_{454}$, as described in [24]. Additionally, the sequences belonging to the wheat homeologous group 4 chromosome arms obtained through Illumina sequencing technology were downloaded from the IWGSC website [21] and are referred as $4 \mathrm{AS}_{\mathrm{I}}, 4 \mathrm{AL}_{\mathrm{I}}, 4 \mathrm{BS}_{\mathrm{I}}, 4 \mathrm{BL}_{\mathrm{I}}, 4 \mathrm{DS}_{\mathrm{I}}$ and $4 \mathrm{DL}_{\mathrm{I}}$. When additional comparisons needed to be done, chromosome arms sequences other than those from the homeologous group 4 were also downloaded from the IWGSC website.

\section{Identification of repetitive elements}

Repetitive sequences were identified using RepeatMasker (RM) [55]. The program inputs were FASTA-formatted archives, whereas the program output consisted of a detailed annotation of the repeats present in the query sequence. Sequence comparisons were performed using the alignment software cross_match (version open-3.3.0) [56].

From the Cross_match output list, the name of the matching interspersed repeat and the class of the repeat were used to classify and count elements belonging to
SMALL RNA, satellites, simple repeats and low complexity regions using a homemade Perl script.

TE interspersed repeat family signatures were identified using Mips-REdat_v9.0p database hosted by the MIPS at PlantsDB [57], that contains $\sim 42.000$ sequences with total length of $\sim 350 \mathrm{Mb}$. The sequences with $>=95 \%$ identity over $>=95 \%$ of its length were considered as redundant and only the longest element from the clusters was used for further analysis.

The repetitive element classification was performed according to hierarchy as suggested by IWGSC [58]: class, subclass and superfamily. DNA transposons were divided into subclasses based on whether they contained terminal inverted repeats (TIRs) or not. RNA retrotransposons were classified as LTR or Non-LTR retrotransposons on the basis of the presence or absence of LTRs.

\section{Identification and annotation of novel LTR retrotransposons}

The scaffolds obtained from $4 \mathrm{DS}_{454}$ and $4 \mathrm{DL}_{454}$ sequences were scanned for LTR retrotransposons using LTR_FINDER [46] and LTR_STRUC [47]. The FASTAformatted scaffolds from the chromosome arm database were used as input data for both programs, whereas the output consisted of putative novel LTR retrotransposon sequences. LTR_FINDER was used with default parameters with the following exceptions: the minimum LTR size was set to 100 and the minimum distance of LTRs (internal domain) was set at $1000 \mathrm{bp}$. The Arabidopsis thaliana (639 tRNAs; Release Feb 2004), Brachypodium distachyon JGI v1.08x (661 tRNAs), Oryza sativa (764 tRNAs), Sorghum bicolor version 1.0 (649 tRNAs) and Zea mays version 4a.53 (1168 tRNAs) databases deposited at Genomic tRNA Database [59] were used to predict the tRNA binding sites typical for LTR structure. tRNA genes prediction was performed using the program tRNAscan-SE [60]. Additional de novo LTR transposons identification was based on sequence homology independent structural features search using LTR_STRUC software [47]. The output candidate LTR retrotransposons were extracted from the scaffolds and manually inspected. Candidate LTR retrotransposons were clustered using CD-HIT (ver. 4.5.7, Jan 32012 [48]). The candidates were further BLAST aligned against MIPS-REdat and manually checked if they belonged to known families, using criteria proposed by Wicker et al. [6]. Two elements belong to the same family if they are at least $80 \%$ identical in at least $80 \%$ of their coding regions and internal domains, or within their LTRs, or in both. The LTRs were aligned using ClustalX [61]. Transposon-associated proteins were identified using BLASTX alignments with NCBI [62] and GyDB [49]. Annotation of LTR retrotransposons was performed according to [6]. The copy number of the candidate LTRs retrotransposons was estimated from alignments with 
survey sequences of all $T$. aestivum chromosome arms deposited at URGI. The alignments showing at least $80 \%$ of identity and at least $80 \%$ coverage after manually inspection were considered positive hits. Additional copies of the novel LTR retrotransposon were searched in the $T$. aestivum (WGS project accession CALP000000000; [50]) and Ae. tauschii (WGS project accession AOCO000000000; [51]) whole genome sequence databases deposited at NCBI.

\section{Estimation of insertion time}

The insertion time of retrotransposons was estimated using the formula $\mathrm{T}=\mathrm{K} / 2 \mathrm{r}$ [63], where $\mathrm{T}, \mathrm{K}$ and $\mathrm{r}$ are time of divergence, average number of substitutions per aligned site and average synonymous substitution rate, respectively. To estimate the divergence time of LTR retrotransposons, $\mathrm{r}$ was set to $1.36 \times 10^{-8}$ substitutions per site per year [64]. The 5'LTR and 3'LTR of each candidate were aligned using ClustalW [61].

\section{Phylogenetic tree construction}

Phylogenetic analyses were conducted in MEGA4 [61]. Aligned sequences were used to generate trees using the Maximum Parsimony method [65]. The bootstrap consensus tree inferred from 500 replicates [66] is taken to represent the evolutionary history of the LTR analyzed [66]. Branches corresponding to partitions reproduced in less than $50 \%$ bootstrap replicates are collapsed. The percentages of replicate trees in which the associated sequence clustered together in the bootstrap test (500 replicates) are shown next to the branches [66]. The MP tree was obtained using the Close-Neighbor-Interchange algorithm with search level $3[65,66]$ in which the initial trees were obtained with the random addition of sequences (10 replicates). All positions containing gaps or missing data were eliminated from the dataset (Complete deletion option).

\section{In situ localization of newly identified and highly abundant repetitive elements}

Here, it was applied fluorescent in situ hybridization labeling in suspension (FISHIS) [42] that uses an additional chromosome specific fluorescent marker which can quantitatively bind to chromosomes. The FISHIS uses microsatellite markers, but only GAA SSR proved to be applicable for reliable chromosome sorting for 12-13 out of 21 bread wheat chromosomes.

Probes for Afa repeat was labeled with digoxigenin (Roche, Mannheim, Germany) and probes for selected microsatellites (4DL_SSR1, 4DL_SSR2, 4BL_SSR1, and 4D_SSR) with Texas Red (Invitrogen, Camarillo, CA, USA) according to [36]. A 260-bp fragment of the Afa family repeat was prepared using PCR with primers AS-A and AS-B on wheat genomic DNA [67]. The SSR repeats labeled by Texas Red were prepared according to [68] using
PCR primers 4DL_SSR1 - $(\mathrm{CAGCG})_{6} /(\mathrm{CGCTG})_{3}$, 4DL_SSR2 - $(\mathrm{CCGTA})_{6} /(\mathrm{TACGG})_{4}, 4 \mathrm{BL} \_\mathrm{SSR} 1$ (CGTAG) $)_{6}$ $(\mathrm{CTACG})_{4}$, 4D_SSR - $(\mathrm{TTACG})_{6} /(\mathrm{CGTAA})_{4}$. The PCR amplification was carried out in a C-1000 Touch $^{\mathrm{Tm}}$ thermal cycler (Bio-Rad, USA) in a volume of $15 \mu \mathrm{l}$ containing $1 \mu \mathrm{mol} / \mathrm{l}$ of each primer, $200 \mu \mathrm{mol} / \mathrm{l}$ of each of the dNTPs, but dATP is supplemented with mixture of dUTP labeled with Texas Red and dATP in ratio $1: 2,1,5 \mathrm{mmol} / \mathrm{l}$ of $\mathrm{MgCl}_{2}, 0,5 \mathrm{U}$ OneTaq DNA Polymerase (New England Biolabs, USA) in supplier recommended buffer. The amplification was done by 40 cycles of $30 \mathrm{sec}$ at $95^{\circ} \mathrm{C}, 30 \mathrm{sec}$ at $60^{\circ} \mathrm{C}$, and elongation was done $30 \mathrm{sec}$ at $72^{\circ} \mathrm{C}$.

Probes for the newly identified transposable elements were labelled directly with Texas red (Invitrogen, Camarillo, CA, USA) using Nick translation approach [69] of PCR product from primers designed for insertion site and internal regions of the transposons. For each transposon two pairs of primers were designed (Additional file 7: Table S6). One of each primers pair was designed directly to the insertion site overlapping host sequence, TSD, and LTR sequence.

The amplicons were designed to be 0,5-4 $\mathrm{kb}$ long. Primers were designed using Primer3 software [70]. The amplification was carried out in a C-1000 Touch $^{\mathrm{Tm}}$ thermal cycler (Bio-Rad, USA) in a volume of $15 \mu \mathrm{l}$ containing $15 \mathrm{ng}$ of Chinese Spring genomic DNA, $1 \mu \mathrm{mol} / \mathrm{l}$ of each primer, $200 \mu \mathrm{mol} / \mathrm{l}$ of each of the dNTPs, $1,5 \mathrm{mmol} / \mathrm{l}$ of $\mathrm{MgCl}_{2}, 0,5$ U OneTaq DNA Polymerase (New England Biolabs, USA) in supplier recommended buffer. The PCR products were separated in $1 \%$ agarose gel. In case of multiple PCR products, the band of expected size was excised from agarose gel, extracted and used for labeling as described above. The identities of the PCR fragments were verified by Sanger sequencing from both corresponding primers.

Chromosome localization of the probes was performed using FISH on wheat metaphase chromosomes (cv. Chinese Spring). Chromosomes were isolated from the meristematic tissue of the root tips treated with ice water for two days and slides were prepared according to [71]. The quality of chromosome spreads was checked under the microscope and the best slides were used for FISH. Post-fixation was performed according to [72].

Hybridization mixture consisting of $40 \%$ formamide, 250 ng of calf thymus DNA, 2x SSC, 15 ng Afa probe, 60 ng transposable element probe and 50\% dextran sulphate up to final $25 \mu \mathrm{l}$ was applied onto the slides. The slides were denatured at $80^{\circ} \mathrm{C}$ for $2.5 \mathrm{~min}$ and incubated in humid chamber at $37^{\circ} \mathrm{C}$ overnight. After the hybridization, slides were stringently washed as described in [73]. The signals of Texas Red labelled probes were observed directly. Digoxigenin-labelled probes were detected using anti-digoxigenin-FITC (Roche, Mannheim, Germany) in the concentration recommended by manufacturer. Chromosome DNA 
was counterstained with 4',6'-diamidino-2-phenylindole (DAPI) in Vectashield (Vector Laboratories, USA).

The preparations were evaluated using Axio Imager Z.2 Zeiss microscope (Zeiss, Oberkochen, Germany) equipped with Cool Cube 1 (Metasystems, Altlussheim, Germany) camera and appropriate filter sets. The capture of fluorescence signals and merging the layers were performed with ISIS software (Metasystems, Germany) and the final image adjustment was done in Adobe Photoshop 6.0.

\section{Availability of supporting data}

The data sets supporting the results of this article are included within the article as Additional file 8 (File 1 and Table S7) that comprises a fasta-formatted file with the nucleotidic sequences of members of the six novel retrotransposon families sequences and table with the main features of each individual sequence. Furthermore, the six novel retrotransposon families will be included in the next update of the Plant Genome and Systems Biology Repeat Element Database (PGSB-REdat).

\section{Additional files}

Additional file 1: Table S1. Satellites identified in the homeologous group 4 chromosome arms from $T$. aestivum.

Additional file 2: Table S2. Microsatellites identified in the homeologous group 4 chromosome arms from $T$. aestivum.

Additional file 3: Table S3. Low complexity elements identified in the homeologous group 4 chromosome arms from T. aestivum.

Additional file 4: Figure S1. Comparison among the size of the scaffolds obtained from 4DS454 and 4DL454 and the contigs obtained from 4DSI and 4DLI. Frequency histogram were constructed showing the sequences size in abscises and the observed frequency of each size in ordinates (JPG extension).

Additional file 5: Table S4. Description of the main features of the members of the new LTR retrotransposon families identified using LTR_FINDER and/or LTR_STRUC.

Additional file 6: Table S5. Novel LTR retrotransposons taxonomy and family members.

Additional file 7: Table S6. PCR primers and conditions to produce FISH probes from the newly identified retrotransposons

Additional file 8: File 1. Contains the fasta-formatted nucleotidic sequences of all the new LTR retrotransposon families' members described in this manuscript (fasta extension). Table S7. Consist of a list of the members of the new LTR retrotransposon families detailing: the bioinformatics methodology that allowed they discovery, complementary structural features and states if they are complete or fragmented elements (Microsoft Word Document).

\section{Competing interests}

The authors declare that they have no competing interests.

\section{Authors' contributions}

IG and JRR have made substantial contributions to conception, design, acquisition of data and analysis and interpretation of results; MV, HV and MK have defined the rationale, the design of the FISH experiments and carried them out; GT, MH, MV, JD, MC and IG have been involved in drafting the manuscript and revising it critically for important intellectual content; VE conceived the study, participated to its design and coordination, and helped to draft the manuscript and revised it critically for important intellectual content. All authors read and approved the final manuscript.

\section{Acknowledgements}

We are grateful to the IWGSC for the access to the chromosome assemblies. This work was supported by the following Institutions: CONICET (International Cooperation Grant Res. 456/ 10/2/2011) ANPCyT (Préstamo BID 2012, PICT 0660), INTA (Res. 418/2012, PNCY01127041) and Universidad Nacional del Sur (PGI-TIR, CSU-142/14).

\section{Author details}

'CERZOS (CCT - CONICET Bahía Blanca) and Universidad Nacional del Sur, Bahía Blanca, Argentina. ${ }^{2}$ Institute of Experimental Botany, Centre of the Region Haná for Biotechnological and Agricultural Research, Šlechtitelů 31, CZ-78371 Olomouc, Czech Republic. ${ }^{3}$ The Genome Analysis Centre (TGAC), Norwich Research Park, Norwich NR4 7UH, UK. ${ }^{4}$ Instituto Recursos Biológicos, Instituto Nacional de Tecnología Agropecuaria (INTA), Hurlingham, Buenos Aires, Argentina. ${ }^{5}$ Estación Experimental Agropecuaria Marcos Juárez, Instituto Nacional de Tecnología Agropecuaria (INTA), Marcos Juárez, Córdoba, Argentina.

Received: 2 January 2015 Accepted: 24 April 2015

Published online: 12 May 2015

\section{References}

1. Petersen G, Seberg O, Yde M, Berthelsen K. Phylogenetic relationships of Triticum and Aegilops and evidence for the origin of the A, B, and D genomes of common wheat (Triticum aestivum). Mol Phylogenet Evol. 2006;39:70-82.

2. Bennetzen JL, Ma J, Devos KM. Mechanisms of recent genome size variation in flowering plants. Ann Bot (Lond). 2005;95:127-32.

3. The International Wheat Genome Sequencing Consortium. A chromosome based draft sequence of the hexaploid bread wheat (Triticum aestivum) genome. Science. 2014;345:1251788.

4. Kidwell MG. Transposable elements and the evolution of genome size in eukaryotes. Genetica. 2002;115:49-63.

5. Rebollo R, Romanish MT, Mager DL. Transposable elements: an abundant and natural source of regulatory sequences for host genes. Annu Rev Genet. 2012:46:21-42.

6. Wicker T, Sabot F, Hua-Van A, Bennetzen JL, Capy P, Chalhoub B, et al. A unified classification system for eukaryotic transposable elements. Nat Rev Genet. 2007;8:973-82.

7. Lerat E. Identifying repeats and transposable elements in sequenced genomes: how to find your way through the dense forest of programs. Heredity. 2010;104:520-33.

8. Ugarković D, Plohl M. Variation in satellite DNA profiles causes and effects. EMBO J. 2002;21:5955-9.

9. Plohl M, Luchetti A, Mestrović N, Mantovani B. Satellite DNAs between selfishness and functionality: structure, genomics and evolution of tandem repeats in centromeric (hetero)chromatin. Gene. 2008:409:72-82.

10. Ananiev EV, Phillips RL, Rines HW. Chromosome-specific molecular organization of maize (Zea mays L.) centromeric regions. Proc Natl Acad Sci U S A. 1998:95:13073-8.

11. Feuillet C, Salse J. Comparative genomics in the Triticeae. In: Feuillet C Muehlbauer GJ, editors. Plant Genetics and Genomics. New York: Springer; 2009. p. 451-77.

12. Beckmann JS, Soller M. Toward a unified approach to genetic-mapping of eukaryotes based on sequence tagged microsatellite sites. BIO-TECHNOLOGY 1990;8:930-2.

13. Gupta PK, Varshney RK. The development and use of microsatellite markers for genetic analysis and plant breeding with emphasis on bread wheat. Euphytica. 2000;113:163-85

14. Waugh R, McLean K, Flavell AJ, Pearce SR, Kumar A, Thomas BB, et al. Genetic distribution of Bare-1-like retrotransposable elements in the barley genome revealed by sequence-specific amplification polymorphisms (S-SAP). Mol Gen Genet. 1997:253:687-94.

15. Flavell AJ, Knox MR, Pearce SR, Ellis TH. Retrotransposon-based insertion polymorphisms (RBIP) for high throughput marker analysis. Plant J. 1998;16:643-50.

16. Kalendar R, Schulman AH. IRAP and REMAP for retrotransposon-based genotyping and fingerprinting. Nat Protoc. 2006;1:2478-84.

17. Luce AC, Sharma A, Mollere OS, Wolfgruber TK, Nagaki K, Jiang J, et al. Precise centromere mapping using a combination of repeat junction markers and chromatin immunoprecipitation-polymerase chain reaction. Genetics. 2006;174:1057-61. 
18. Devos KM, Ma J, Pontaroli AC, Pratt LH, Bennetzen JL. Analysis and mapping of randomly chosen bacterial artificial chromosome clones from hexaploid bread wheat. Proc Natl Acad Sci U S A. 2005;102:19243-8.

19. Paux E, Roger D, Badaeva E, Gay G, Bernard M, Sourdille $P$, et al. Characterizing the composition and evolution of homeologous genomes in hexaploid wheat through BAC-end sequencing on chromosome 3B. Plant J. 2006;48:463-74.

20. Wanjugi H, Coleman-Derr D, Huo NX, Kianian SF, Luo MC, Wu JJ, et al. Rapid development of PCR-based genome-specific repetitive DNA junction markers in wheat. Genome. 2009:52:576-87.

21. International Wheat Genome Sequencing Consortium at www.wheatgenome.org

22. Doležel J, Simkova H, Kubalakova M, Safar J, Suchankova P, Cihalikova J, et al. Chromosome genomics in the Triticeae. In: Feuillet C, Muehlbauer GJ, editors. Plant Genetics and Genomics. New York: Springer; 2009. p. 285-316.

23. Doležel J, Kubaláková M, Paux E, Bartoš J, Feuillet C. Chromosome-based genomics in the cereals. Chromosom Res. 2007;15:51-66.

24. Helguera M, Rivarola M, Clavijo B, Marthis M, Vanzetti L, González S, et al. Sequence of chromosome 4D of bread wheat reveals its structure and virtual gene order. Plant Sci. 2015;233:200-12.

25. Ling HQ, Zhao S, Liu D, Wang J, Sun H, Zhang C, et al. Draft genome of the wheat A-genome progenitor Triticum urartu. Nature. 2013;496:87-90.

26. Jia J, Zhao S, Kong X, Li Y, Zhao G, He W, et al. Aegilops tauschii draft genome sequence reveals a gene repertoire for wheat adaptation. Nature. 2013;496:91-5.

27. Vitulo N, Albiero A, Forcato C, Campagna D, Dal Pero F, Bagnaresi P, et al. First Survey of the wheat chromosome $5 \mathrm{~A}$ composition through a next generation sequencing approach. PLoS One. 2011;6(10):e26421.

28. Sehgal SK, Li W, Rabinowicz PD, Chan A, Simková H, Doležel J, et al. Chromosome arm-specific BAC end sequences permit comparative analysis of homoeologous chromosomes and genomes of polyploid wheat. BMC Plant Biology. 2012;12:64.

29. Hernandez P, Martis M, Dorado G, Pfeifer M, Gálvez S, Schaaf S, et al. Next generation sequencing and syntenic integration of flow sorted arms of wheat chromosome $4 \mathrm{~A}$ exposes the chromosome structure and gene content. Plant J. 2012;69:377-86.

30. Tanaka T, Kobayashi F, Joshi GP, Onuki R, Sakai H, Kanamori H, et al. Next-Generation Survey Sequencing and the Molecular Organization of Wheat Chromosome 6B. DNA Res. 2014:21:103-14.

31. Sergeeva EM, Afonnikov DA, Koltunova MK, Gusev VD, Miroshnichenko LA, Vrána J, et al. Common wheat chromosome 5B composition analysis using low-coverage 454 Sequencing. The Plant Genome. 2014;7:1-16.

32. Metzker ML. Sequencing technologies - the next generation. Nat Rev Genet. 2010;11:31-46.

33. Kurtz S, Narechania A, Stein JC, Ware D. A new method to compute K-mer frequencies and its application to annotate large repetitive plant genomes. BMC Genomics. 2008;9:517

34. Mayer KFX, Taudien S, Martis M, Šimková H, Suchánková P, Gundlach H, et al. Gene content and virtual gene order of barley chromosome $1 \mathrm{H}$. Plant Physiol. 2009;151:496-505.

35. Oyola SO, Otto TD, Gu Y, Maslen G, Manske M, Campino S, et al. Optimizing Illumina next-generation sequencing library preparation for extremely AT biased genomes. BMC Genomics. 2012;13:1.

36. Kubaláková M, Kovářová $P$, Suchánková P, Číhalíková J, Bartoš J, Lucretti S, et al. Chromosome sorting in tetraploid wheat and its potential for genome analysis. Genetics. 2005;170:823-9.

37. Morgante M, Hanafey M, Powell W. Microsatellites are preferentially associated with nonrepetitive DNA in plant genomes. Nat Genet. 2002:30:194-200

38. Huo N, Lazo GR, Vogel JP, You FM, Ma Y, Hayden DM, et al. The nuclear genome of Brachypodium distachyon: analysis of BAC end sequences. Funct Integr Genomics. 2008:8:135-47.

39. Röder MS, Korzun V, Wandehake K, Planschke J, Tixier MH, Leroy P, et al. A microsatellite map of wheat. Genetics. 1998;149:2007-23.

40. Pestsova E, Ganal MW, Röder MS. Isolation and mapping of microsatellite markers specific for the D genome of bread wheat. Genome. 2000;43:689-97.

41. Vrána J, Kubaláková M, Šimková H, Číhalíková J, Lysák MA, Doležel J. Flow-sorting of mitotic chromosomes in common wheat (Triticum aestivum L.). Genetics. 2000;156:2033-41.

42. Giorgi D, Farina A, Grosso V, Gennaro A, Ceoloni C, Lucretti S. FISHIS: fluorescence in situ hybridization in suspension and chromosome flow sorting made easy. PLoS One. 2013;8:e57994.
43. Cuadrado A, Cardoso M, Jouve N. Physical organisation of simple sequence repeats (SSRs) in Triticeae: structural, functional and evolutionary implications. Cytogenet Genome Res. 2008;120:210-9.

44. Witte $C P$, Le $\mathrm{QH}$, Bureau T, Kumar A. Terminal-repeat retrotransposons in miniature (TRIM) are involved in restructuring plant genomes. Proc Natl Acad Sci U S A. 2001:98:13778-83.

45. Kalendar R, Vicient CM, Peleg O, Anamthawat-Jonsson K, Bolshoy A, Schulman A. Large retrotransposon derivatives: abundant, conserved but nonautonomous retroelements of barley and related genomes. Genetics. 2004;166:1437-50.

46. Xu Z, Wang H. LTR_FINDER: an efficient tool for the prediction of full-length LTR retrotransposons. Nucleic Acids Res. 2007:35(Web Server issue):W265-W26.

47. McCarthy EM, MCDonald JF. LTR_STRUC: a novel search and identification program for LTR retrotransposons. Bioinformatics. 2003;19:362-7.

48. CD-HIT Suite: Biological Sequence Clustering and Comparison. http://weizhong-lab.ucsd.edu/cdhit_suite/cgi-bin/index.cgi?cmd=h-cd-hit-est.

49. Gypsy Database. www.gydb.org.

50. Brenchley R, Spannagl M, Pfeifer M, Barker GL, D'Amore R, Allen AM, et al. Analysis of the bread wheat genome using whole-genome shotgun sequencing. Nature. 2012;491:705-10

51. Marcussen T, Sandve SR, Heier L, Spannagl M, Pfeifer M, International Wheat Genome Sequencing Consortium, et al. Ancient hybridizations among the ancestral genomes of bread wheat. Science. 2014:345:6194.

52. Cordaux R, Batzer MA. The impact of retrotransposons on human genome evolution. Nat Rev Genet. 2009;10:691-703.

53. Sharma A, Presting GG. Centromeric retrotransposon lineages predate the maize/rice divergence and differ in abundance and activity. Mol Genet Genomics. 2008;279:133-47.

54. Neumann P, Navrátilová A, Kobližková A, Kejnovský E, Hřibová E, Hobza R, et al. Plant centromeric retrotransposons: a structural and cytogenetic perspective. Mob DNA. 2011;2:4

55. Repeatmasker. www.repeatmasker.org

56. Bedell JA, Korf I, Gish W. MaskerAid: a performance enhancement to RepeatMasker. Bioinformatics. 2000;16:1040-1.

57. MIPS Plant DB. ftp://ftpmips.helmholtz-muenchen.de/plants/REdat/.

58. Guidelines for Annotating Wheat Genomic Sequences. http://wheat.pw.usda.gov/ ITMI/Repeats/gene_annotation.pdf.

59. Genomic tRNa database. http://lowelab.ucsc.edu/GtRNAdb/.

60. Lowe TM, Eddy SR. tRNA scan-SE: a program for improved detection of transfer RNA genes in genomic sequence. Nucleic Acids Res. 1997;25:955-64

61. Larkin MA, Blackshields G, Brown NP, Chenna R, McGettigan PA, McWilliam H, et al. ClustalW and ClustalX version 2. Bioinformatics. 2007:23:2947-8.

62. Altschul SF, Gish W, Miller W, Myers EW, Lipman DJ. Basic local alignment search tool. J Mol Biol. 1990:215:403-10.

63. SanMiguel P, Gaut BS, Tikhonov A, Nakajima Y, Bennetzen JL. The paleontology of intergene retrotransposons of maize. Nature. 1998;20:43-5.

64. Ma J, Bennetzen JL. Rapid recent growth and divergence of rice nuclear genomes. Proc Natl Acad Sci U S A. 2004;101:12404-10.

65. Eck RV, Dayhoff MO. Atlas of Protein Sequence and Structure, National Biomedical Research Foundation. Maryland: Silver Springs; 1966.

66. Felsenstein J. Confidence limits on phylogenies: an approach using the bootstrap. Evolution. 1985;39:783-91.

67. Nagaki K, Tsujimoto H, Isono K, Sasakuma T. Molecular characterization of a tandem repeat, Afa family, and its distribution among Triticeae. Genome. 1995:38:479-86.

68. Vrána J, Šimková H, Kubaláková M, Číhalíková J, Doležel J. Flow cytometric chromosome sorting in plants: The next generation. Methods. 2012:57:331-7.

69. Kato A, Albert PS, Vega JM, Bichler JA. Sensitive fluorescence in situ hybridization signal detection in maize using directly labelled probes produced by high concentration DNA polymerase nick translation. Biotech Histochem. 2006;81:71-8.

70. Primer 3 Software. http://bioinfo.ut.ee/primer3-0.4.0/.

71. Masoudi-Nejad A, Nasuda S, McIntosh RA, Endo TR. Transfer of rye chromosome segments to wheat by a gametocidal system. Chromosome Res. 2002;10:349-57.

72. Ma L, Xiao Y, Huang H, Wang QW, Rao WN, Feng Y, et al. Direct determination of molecular haplotypes by chromosome microdissection. Nat Methods. 2010;7:299-301. 
73. Kubaláková M, Valárik M, Bartoš J, Vrána J, Cíhalíková J, Molnár-Láng M, et al. Analysis and sorting of rye (Secale cereale L.) chromosomes using flow cytometry. Genome. 2003:46:893-905.

74. Šafár̆ J, Šimková H, Kubaláková M, Číhalíková J, Suchánková P, Bartoš J, et al. Development of chromosome-specific BAC resources for genomics of bread wheat. Cytogenet Genome Res. 2010;129:211-23.

75. Tamura K, Dudley J, Nei M, Kumar S. MEGA4: Molecular Evolutionary Genetics Analysis (MEGA) software version 4.0. Mol Biol Evol. 2007;24:1596-9.

\section{Submit your next manuscript to BioMed Central} and take full advantage of:

- Convenient online submission

- Thorough peer review

- No space constraints or color figure charges

- Immediate publication on acceptance

- Inclusion in PubMed, CAS, Scopus and Google Scholar

- Research which is freely available for redistribution 